\title{
The Capacity of Ad Hoc Networks with Heterogeneous Traffic Using Cooperation
}

\author{
Mingyue $\mathrm{Ji}^{\dagger}$, Zheng $\mathrm{Wang}^{\dagger}$, Hamid R. Sadjadpour ${ }^{\dagger}$, J.J. Garcia-Luna-Aceves ${ }^{\ddagger}$ \\ Department of Electrical Engineering ${ }^{\dagger}$ and Computer Engineering ${ }^{\ddagger}$ \\ University of California, Santa Cruz, 1156 High Street, Santa Cruz, CA 95064, USA \\ $\ddagger$ Palo Alto Research Center (PARC), 3333 Coyote Hill Road, Palo Alto, CA 94304, USA \\ Email:\{davidjmy, wzgold, hamid, jj\}@ soe.ucsc.edu
}

\begin{abstract}
We study the scaling laws for wireless ad hoc networks in which the distribution of $n$ nodes in the network is homogeneous but the traffic they carry is heterogeneous. More specifically, we consider the case in which a given node is the data-gathering sink for $k$ sources sending different information to it, while the rest of the $s=n-k$ nodes participate in unicast sessions with random destinations chosen uniformly. We present a separation theorem for heterogeneous traffic showing that the optimum order throughput capacity can be attained in a wireless network in which traffic classes are distributed uniformly by endowing each node with multiple radios, each operating in a separate orthogonal channel, and by allocating a radio per node to each traffic class. Based on this theorem, we show how this order capacity can be attained for the unicast and data-gathering traffic classes by extending cooperative communication schemes that have been proposed previously.
\end{abstract}

\section{INTRODUCTION}

The scaling laws of wireless networks with homogeneous traffic and uniform node distribution have been extensively studied in the literature. Gupta and Kumar [1] evaluated the capacity of wireless networks with uniform traffic and showed that the capacity scales as $\Theta\left(\sqrt{\frac{n}{\log n}}\right)$ according to the protocol model ${ }^{1}$. This result was achieved by considering no cooperation among nodes with simple point-to-point communication. Xie and Kumar [2], [3] subsequently investigated the information-theoretic capacity of wireless networks with cooperation among different nodes in the extended network. The achievable capacity with cooperation for dense networks was studied by Özgür et al. in [4].

Only a handful of prior works investigate heterogeneous traffic in the network. Keshavarz-Haddad et al. [5] introduced the concept of transmission arena. Based on that definition, they presented a method to compute the upper bound of the capacity for different traffic patterns and different topologies of the network. However, they did not provide closed-form scaling laws for the network capacity. Toumpis [6] investigated the throughput capacity when there are $s$ sources and $s^{\varepsilon}$ destinations in the network, where $0<\varepsilon<1$. Liu et al. [7]

\footnotetext{
${ }^{1}$ Given two functions $f$ and $g$, we say that: 1) $f(n)=O(g(n))$ if there exists a constant $c$ and integer $N$ such that $f(n) \leq c g(n)$ for $n>N$. 2) $f(n)=o(g(n))$ if $\lim _{n \rightarrow \infty} \frac{f(n)}{g(n)}=0$. 3) $f(n)=\Omega(g(n))$ if $g(n)=$ $O(f(n))$. 4) $f(n)=\omega(g(n))$ if $g(n)=o(f(n))$. 5) $f(n)=\Theta(g(n))$ if $f(n)=O(g(n))$ and $g(n)=O(f(n))$.
}

extended this result by relaxing the constraint on the number of sources and destinations. While these results [6], [7] address asymmetric traffic, the results apply to the case of a single type of traffic pattern in the network.

We consider heterogeneous traffic in the network supporting different types of traffic patterns. In particular, we consider two types of traffic classes, namely, data-gathering traffic in which one of the nodes in the network acts as a sink with many sources transmitting different packets to that node, and the rest of the nodes in the network participate in unicast traffic flows. The distribution of nodes in the network is still uniform. To the best of our knowledge, this heterogeneous traffic model has not been studied in the literature, except our own preliminary work [8], which assumes that the bandwidth assignment is proportional to the traffic for each cell, does not consider cooperation among nodes, and the capacity analysis is based on the protocol model [1]. There is some prior work addressing data gathering [9], [10], [11] as the only type of traffic in a network. Rodoplu et al. [12], [13] computed the network capacity for data gathering and unicast flows separately by utilizing game theory and defining a new capacity concept named core capacity.

This paper introduces a new approach to support heterogeneous traffic efficiently in a wireless network by dividing the available bandwidth into multiple channels separated in frequency and allocated dynamically to specific traffic classes consisting of the aggregation of one or more flows. We present a separation theorem showing that, in a multiplechannel multiple-radio wireless network, the optimum order throughput capacity can be attained in a wireless network in which traffic classes are distributed uniformly by allocating a radio per node to each traffic class. Based on this theorem, we extend the Three-Phase approach first introduced by Özgür et. al. [4] to accomodate different traffic classes. We demonstrate that the maximum per-node throughput capacity of $\Theta(\log (n))$ for unicast traffic can be attained. This capacity was provided as an upper bound originally by Özgür et al [4] without providing any specific communication scheme.

The rest of the paper is organized as follows. Section II presents important assumptions and definitions used in our analysis. Section III presents an upper bound on the throughput capacity of a wireless network. Section IV presents the Three- 
Phase scheme for different operating regions of the network. The achievable aggregate throughput for the heterogeneous traffic is also presented. Section $\mathrm{V}$ discusses the implications of our work and future research directions.

\section{Wireless Network MOdel}

We consider a network with nodes uniformly distributed in a dense network with constant area $A$. For the data-gathering traffic, a single node, called the access point, is the destination for $k$ sources in the network. For the rest of the $s=n-$ $k$ nodes in the network, source-destination pairs are selected randomly and uniformly. For each source node $s_{i}$, where $i=$ $1, \cdots, n$, the data rate is denoted by $R_{s_{i}}(n)$. Similarly, for each destination node $d_{j}$, where $j=1, \cdots, s+1$, the rate is denoted by $R_{d_{j}}(n)$. The capacity of the network is defined as

$$
\begin{array}{r}
C(n)=\max _{R_{s_{i}} \text { and } R_{d_{j}} ; i=1, \cdot, n ; j=1, \cdot, s+1} \\
\left(\min \left(\sum_{i=1}^{n} R_{s_{i}}(n), \sum_{j=1}^{s+1} R_{d_{j}}(n)\right)\right)
\end{array}
$$

The total bandwidth in the network is $W$ hertz. Furthermore, the total power for transmission in the network is assumed to be $P$. The complex channel gain between nodes $i$ and $k$ at time $m$ is given by $H_{i k}[m]=\left(\sqrt{G} \exp \left(j \theta_{i k}[m]\right)\right) /\left(d_{i k}^{\frac{\alpha}{2}}\right)$, where $d_{i k}$ is the distance between nodes $i$ and $k, \theta_{i k}[m]$ is the random phase at time $m$ which is uniformly distributed between $[0,2 \pi]$. For all pairs of $i$ and $k, \theta_{i k}[\mathrm{~m}] s$ are independent and identically distributed (i.i.d.) random variables. Note that $\theta_{i k}[m]$ and $d_{i k}$ are assumed to be independent. The parameters $G$ and $\alpha>2$ are constants. The channel parameter is always known to the receiver during transmission and the phase is a fast fading ${ }^{2}$. The received signal by node $i$ at time $m$ is given by $Y_{i}=\sum_{k=1}^{n} H_{i k}[m] X_{k}[m]+Z_{i}[m]$, where $X_{k}[m]$ is the transmitted signal by node $k$ at time $m$ and $Z_{i}[m]$ is white circularly symmetric Gaussian noise of variance $N_{0}$. The notations $K_{i}$ and $C_{i}$ for any integer $i$ represent constant values.

\section{An Upper Bound ON The Network CAPACITY}

The information-theoretic upper bound of the aggregate throughput in wireless networks is derived. This upper bound is compared subsequently with the achievable lower bound to demonstrate the effectiveness of the routing strategies utilized in this paper.

Theorem 3.1: In a wireless network with $k$ sources for the access node and $s$ source-destination pairs where $s+k=n$, the capacity is upper bounded by

$$
C(n) \leq K_{1}(s+1) \log n
$$

where $K_{1}$ is a constant.

\footnotetext{
${ }^{2}$ Our channel assumption is identical to the one used by Özgür et al. [4] and the reader can read the detailed justification for this channel model in [4].
}

Proof: It is clear from Eq. (1) that

$$
C(n) \leq \max _{R_{d_{j}} ; j=1, \cdot, s+1}\left(\sum_{j=1}^{s+1} R_{d_{j}}(n)\right)
$$

For any arbitrary destination $j$, the network is equivalent of a multiple-input single-output channel with upper bound given by ( [14], Eq. (5.31))

$$
R_{d_{j}}(n) \leq \log \left(1+\frac{P}{N_{0}} \sum_{k=1, k \neq j}^{k=n} \frac{G}{d_{k j}^{\alpha}}\right) .
$$

Özgür et al. [4] showed that

$$
\operatorname{Pr}\left(d_{\min }<\frac{1}{n^{1+\delta}}\right) \leq n\left(1-\left(1-\frac{\pi}{n^{2+2 \delta}}\right)^{n-1}\right) .
$$

This probability goes to zero as $n$ tends to infinity, which means that the distance between any two nodes is at least $\frac{1}{n^{1+\delta}}$. Accordingly, Eq. (3) becomes

$$
\begin{aligned}
C(n) & \leq(s+1) \log \left(1+\frac{P G}{N_{0}} n^{\alpha(1+\delta)+1}\right) \\
& =K_{1}(s+1) \log n
\end{aligned}
$$

From Theorem 3.1, we observe that the upper bound of the capacity scales as $\Theta((s+1) \log n)$.

\section{Main Results}

We first provide a separation theorem for heterogeneous traffic in wireless networks. Then, the achievable rate for this network model is presented.

Theorem 4.1: Separation Theorem for Heterogeneous Traffic in Wireless Networks: Consider a network with total available bandwidth $W$ in which $k_{1}$ traffic classes of equal priority are distributed uniformly in the network with each traffic class utilizing $\frac{W}{k_{1}}$ bandwidth. Separating the traffic classes and using a separate frequency and radio per node for each traffic class provides the optimum aggregate order capacity for the network.

Proof: Let $n$ be the total number of nodes in the network and for each traffic class $T_{i}, i=1, \ldots, k_{1}$ let there be $n_{i}$ nodes as sources or destinations for this traffic class, where $n_{i} \leq n$. Assume that the optimum routing communication scheme is used for this traffic class. If only the $n_{i}$ source and destination nodes were used in the network for this traffic class, one can design the optimum technique to achieve the maximum throughput capacity for this traffic class. It is well known that adding relays in a network facilitates the transmission of information and can increase its capacity [9], [10]. Now, by utilizing the remaining $n-n_{i}$ nodes in the network as relays for the traffic class $T_{i}$, at least the same capacity can be attained for the traffic class as when there were no relays in the network for the traffic class. However, one can design a new optimum technique to achieve the maximum throughput capacity considering the relays which is at least equal to or greater than the previous case. Therefore, for any traffic class 
utilizing a multiple-channel multiple-radio (MC-MR) system provides an upper bound on the capacity of that traffic class.

In addition, given that the total bandwidth of the network $W$ and the number of traffic classes $k_{1}$ are constants, dividing the bandwidth into smaller portions does not change the order throughput capacity and the optimality of the results. Hence, MC-MR systems provide the optimal order capacity for heterogeneous traffic networks.

This simple theorem provides a significant result for heterogeneous traffic networks. The theorem states that, by utilizing MC-MR systems, the nodes in the network that are not sources or destinations of a particular traffic class can be used as relays to improve the capacity of the network. We will show some examples of this intuitive result in this paper.

Another important implication of the above theorem is the fact that all analysis for homogeneous traffic can be used for heterogeneous traffic, as long as we allow some nodes in the network to participate as relays. Then the capacity of the network can be computed for that particular traffic pattern by changing the number of relays.

The following theorem establishes the main contribution of this paper, and the rest of the paper is dedicated to proving this theorem.

Theorem 4.2: Consider a network with one access node receiving information from $k$ sources and $s$ different nodes that select random destinations uniformly from all other nodes in the network. By using the MC-MR scheme, the achievable aggregate throughput is given by

$$
\begin{aligned}
& R(n)= \\
& \begin{cases}\Omega((s+1) \log n), & s=O\left(\left(\frac{n}{\log n}\right)^{\frac{1}{2+\varepsilon_{2}+\varepsilon_{3}}}\right), \\
\Omega\left(\frac{n}{s^{1+\varepsilon_{2}+\varepsilon_{3}}}+\log n\right), & \Omega\left(\left(\frac{n}{\log n}\right)^{\frac{1}{2+\varepsilon_{2}+\varepsilon_{3}}}\right)=s \\
& =O\left(\left(\frac{n \log n}{\log \log n}\right)^{\frac{1}{2+\varepsilon_{2}+\varepsilon_{3}}}\right), \\
\Omega\left(\frac{s \log \log s}{\log s}+\log n\right), & \Omega\left(\left(\frac{n \log n}{\log \log n}\right)^{\frac{1}{2+\varepsilon_{2}+\varepsilon_{3}}}\right)=s \\
& =O\left(\left(\frac{n \log n}{\log \log n}\right)^{\frac{1}{2}}\right), \\
\Omega\left(\frac{n}{s}+\log n\right), & \Omega\left(\left(\frac{n \log n}{\log \log n}\right)^{\frac{1}{2}}\right)=s \\
\Omega\left(s^{1-\varepsilon_{1}+\varepsilon_{4}}+\log n\right), & \left.=O\left(n^{\frac{1}{2-\varepsilon_{1}+\varepsilon_{4}}}\right)^{\frac{1}{2-\varepsilon_{1}+\varepsilon_{4}}}\right)=s \\
& =O(n)\end{cases}
\end{aligned}
$$

where $\varepsilon_{1}, \varepsilon_{2}, \varepsilon_{3}$, and $\varepsilon_{4}$ are positive small numbers between 0 and 1 and $\varepsilon_{4}<\varepsilon_{1}$.

Note that there are two terms corresponding to unicast and data gathering communications for each capacity region. For example, in the first capacity region of Eq. (7), the terms $s \log n$ and $\log n$ are related to unicast and data-gathering communication, respectively. It can be easily shown that the per-node throughput capacity of the unicast communication for our technique is always greater than that of [4], while the total unicast capacity is smaller than that of [4], because there are only $s<n$ unicast pairs in our scheme. The reason for this capacity improvement is the use of relays in our scheme. We also note that in [4] all nodes were participating in unicast communication, while in [9], there are few unicast sessions and the rest of the nodes are relays. One of the results in this paper is the computation of the throughput capacity using MIMO cooperation when the number of relays in the network changes as a function of $n$. We also observe that, for the first capacity region, the throughput capacity is equal to the upper bound capacity that was derived in Section III. This is the first paper to report per node throughput of $\log n$ for unicast communications.

\section{A. Capacity Analysis for Unicast Traffic}

Based on the separation theorem, it is sufficient to derive the throughput capacity for each traffic class independently without being concerned about the optimality of our result. Our main approach for the computation of unicast traffic capacity is based on the hierarchical MIMO cooperation approach introduced by Özgür et al. [4]. However, given that we take advantage of relays in this paper, we modify the ThreePhase scheme in [4] based on the number of relays available in the network in order to maximize the achievable capacity. The details of these schemes are described in the rest of this section.

1) The Case of: $s=O\left(\left(\frac{n}{\log n}\right)^{\frac{1}{2+\varepsilon_{2}+\varepsilon_{3}}}\right)$

First, we introduce a useful lemma from [7].

Lemma 4.3: Let $B(m, n)$ be the random variable that counts the maximum number of balls in any bin when we throw $m$ balls independently and uniformly at random into $n$ bins. Then

$$
B(m, n)=\left\{\begin{array}{lr}
\Theta\left(\frac{\log n}{\log \frac{n}{m}}\right), & \text { if } m<\frac{n}{\log n}, \\
\Theta\left(\frac{\log n}{\left.\log \frac{n \log n}{m}\right),},\right. & \text { if } \frac{n}{\text { poly } \log n} \leq m \ll n \log n, \\
\Theta(\log n), & \text { if } m=c \cdot n \log n \\
\Theta\left(\frac{m}{n}\right), & \text { for some constant } c, \\
\text { if } m \gg n \log n .
\end{array}\right.
$$

By dividing the network into $s^{1+\varepsilon_{2}}$ clusters and using Lemma 4.3, the following theorem can be proved.

Theorem 4.4: Consider a network with $n$ nodes and $s$ source nodes distributed uniformly in the network such that $s=n^{\alpha_{1}}$ and $0 \leq \alpha_{1}<\frac{1}{1+\varepsilon_{2}+\varepsilon_{3}}$. If the network is divided into $s^{1+\varepsilon_{2}}$ clusters, there are at most $\Theta(1)$ source nodes and $\Theta\left(\frac{n}{s^{1+\varepsilon_{2}}}\right)$ nodes w.h.p. in each cluster. In any circle with 
radius $\frac{\sqrt{A}}{2 s \frac{1+\varepsilon_{2}+\varepsilon_{3}}{2}}$ or $\frac{\sqrt{A}}{2 n \frac{\left(1+\varepsilon_{2}\right) \beta_{1}}{2}}$, where $0<\beta_{1}<1$, there are $\Theta\left(\frac{n}{s^{1+\varepsilon_{2}+\varepsilon_{3}}}\right)$ or $\Theta\left(\frac{n}{n^{\left(1+\varepsilon_{2}\right) \beta_{1}}}\right)$ nodes, respectively w.h.p.

The proof of this theorem is given in Appendix A. To simplify the analysis, we assume that there are exactly $K_{2} \frac{n}{s^{1+\varepsilon_{2}+\varepsilon_{3}}}$ nodes in each circle with radius $\frac{\sqrt{A}}{2 s \frac{1+\varepsilon_{2}+\varepsilon_{3}}{\sqrt{A}^{2}}}, K_{2} \frac{n}{n^{\left(1+\varepsilon_{2}\right) \beta_{1}}}$ nodes in each circle with radius $\frac{\sqrt{A}}{2 n \frac{\left(1+\varepsilon_{2}\right) \beta_{1}}{2}}$, and $K_{3}$ source nodes in each cluster. We now introduce our Three-Phase communication scheme for each capacity region. Without loss of generality, the source nodes are considered at the center of each cluster to simplify the analysis. The total transmit power required to transmit all unicast traffic is $P_{1}$ Watts and for each phase is $P_{1}^{\prime}=\frac{1}{3} P_{1}$.

Phase 1. Distribution of packets from source to relays in the same cluster: As in previous work [4], we divide the entire network into smaller cells or clusters of square shape. If the network area is divided into $s^{1+\varepsilon_{2}}$ clusters, then each cluster has an area of $\frac{A}{s^{1+\varepsilon_{2}}}$. In order to avoid interference, the cells are grouped into $K_{6}$ non-interfering groups using a TDMA scheme. We divide this region into two regions of $s=O\left(n^{\beta_{1}}\right)$ and $\Omega\left(n^{\beta_{1}}\right)=s=O\left(\left(\frac{n}{\log n}\right)^{\frac{1}{2+\varepsilon_{2}+\varepsilon_{3}}}\right)$ where $\beta_{1}$ is an arbitrarily small constant number. Note that there are at most $K_{3}$ sources in each cluster. When $\Omega\left(n^{\beta_{1}}\right)=s=$ $O\left(\left(\frac{n}{\log n}\right)^{\frac{1}{2+\varepsilon_{2}+\varepsilon_{3}}}\right)$, then we let $K_{2} \frac{n}{s^{1+\varepsilon_{2}+\varepsilon_{3}}}$ nodes in the circle of radius $\frac{\sqrt{A}}{2 s \frac{1+\varepsilon_{2}+\varepsilon_{3}}{2}}$ help each source in the cluster to transmit information. For $s=O\left(n^{\beta_{1}}\right)$, it is easy to show that $K_{2} \frac{n}{s^{1+\varepsilon_{2}}}>K_{2} \frac{n}{n^{\left(1+\varepsilon_{2}\right) \beta_{1}}}$. Therefore, only $K_{2} \frac{n}{n^{\left(1+\varepsilon_{2}\right) \beta_{1}}}$ relay nodes in the circle of radius $\frac{\sqrt{A}}{2 n \frac{\left(1+\varepsilon_{2}\right) \beta_{1}}{2}}$ help each source in the cluster to transmit information. These nodes operate as relays in the network. Each source node transmits $K_{2} \frac{n}{s^{1+\varepsilon_{2}+\varepsilon_{3}}}$ ( or $K_{2} \frac{n}{n^{\left(1+\varepsilon_{2}\right) \beta_{1}}}$ )blocks of information based on the size of $s$ to the relays in its cluster. Each block has a length of $L$. At the end of phase 1, each relay in the circle has received a different block of information. The next Theorem describes the total aggregate throughput in the network.

Theorem 4.5: Consider a network organized into $s^{1+\varepsilon_{2}}$ clusters. Then by implementing the TDMA scheme described above when $s=O\left(\left(\frac{n}{\log n}\right)^{\frac{1}{2+\varepsilon_{2}+\varepsilon_{3}}}\right)$, the aggregate throughput for the network is $\frac{s\left(K_{4} \log n+K_{5}\right)}{K_{6}}$.

The proof of this theorem is given in Appendix B.

When $\Omega\left(n^{\beta_{1}}\right)=s=O\left(\left(\frac{n}{\log n}\right)^{\frac{1}{2+\varepsilon_{2}+\varepsilon_{3}}}\right)$, then the transmission time required to complete this phase is

$$
t_{\text {Phase 1 }}=\frac{s K_{2} L \frac{n}{s^{1+\varepsilon_{2}+\varepsilon_{3}}}}{\frac{s\left(K_{4} \log n+K_{5}\right)}{K_{6}}}=\frac{K_{6} K_{2} L \frac{n}{s^{1+\varepsilon_{2}+\varepsilon_{3}}}}{K_{4} \log n+K_{5}} .
$$

When $s=O\left(n^{\beta_{1}}\right)$, then the transmission time required to complete this phase is

$$
t_{\text {Phase 1 }}=\frac{s K_{2} L \frac{n}{n^{\left(1+\varepsilon_{2}\right) \beta_{1}}}}{\frac{s\left(K_{4} \log n+K_{5}\right)}{K_{6}}}=\frac{K_{6} K_{2} L \frac{n}{n^{\left(1+\varepsilon_{2}\right) \beta_{1}}}}{K_{4} \log n+K_{5}} .
$$

Phase 2. MIMO Cooperation Transmission: At the beginning of the second phase, all nodes in the cluster containing the source nodes decode the information into a finite number of bits. This information is mapped into $C_{1} \frac{n}{s^{1+\varepsilon_{2}+\varepsilon_{3}}}$ (or $C_{1} \frac{n}{n^{\left(1+\varepsilon_{2}\right) \beta_{1}}}$ when $s=O\left(n^{\beta_{1}}\right)$ ) symbols, where $C_{1}$ is a constant. Then the source nodes along with the relays form a distributed MIMO system to transmit their information to the destinations and the relays surrounding the destinations in that cluster (see Fig. 1). Given that there are $s$ sources in the network, there are $s$ MIMO transmissions to complete this phase.

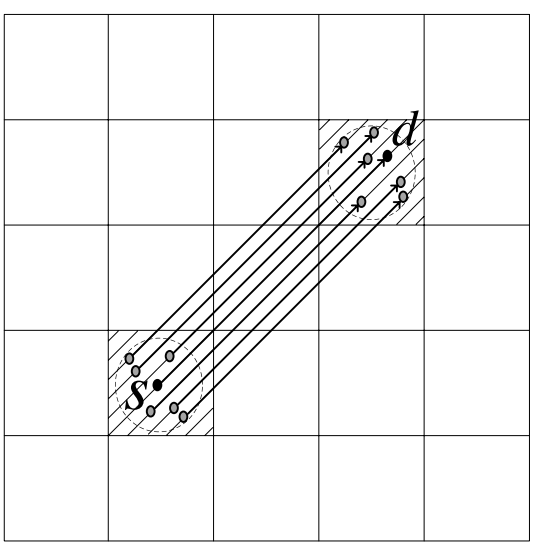

Fig. 1. MIMO Cooperation Transmission. The black nodes represent sources or destinations. The gray nodes are the relays.

The aggregate throughput for this phase is given by the following lemma.

Lemma 4.6: The aggregate throughput for the MIMO cooperation transmission scheme is at least $K_{7} \frac{n}{s^{1+\epsilon_{2}+\epsilon_{3}}}$ when $\Omega\left(n^{\beta_{1}}\right)=s=O\left(\left(\frac{n}{\log n}\right)^{\frac{1}{2+\varepsilon_{2}+\varepsilon_{3}}}\right)$ and $K_{7} \frac{n}{n^{\left(1+\varepsilon_{2}\right) \beta_{1}}}$ when $s=O\left(n^{\beta_{1}}\right)$ for the MIMO quantized channel.

This lemma is proved in [4]. It is easy to show that the total required time for Phase 2 is $t_{\text {Phase } 2}=\Theta(s)=\frac{C_{1}}{K_{7}} s$.

Phase 3. Transmission from Relays to Destination: Phase 3 is the reverse of phase 1 with relays in the destination cluster quantizing the observed information and transmitting them sequentially to the destination. Using Lemma 4.6 it can be proved [4] that $K_{7} \frac{n}{s^{1+\varepsilon_{2}+\varepsilon_{3}}}\left(\right.$ or $K_{7} \frac{n}{n^{\left(1+\varepsilon_{2}\right) \beta_{1}}}$ for $s=O\left(n^{\beta_{1}}\right)$ ) throughput can be achieved. Note that the TDMA scheme for parallel transmissions in clusters is implemented for Phase 3.

From the above discussion, the time requirement for Phase 3 , the total time and the aggregate throughput as the result of three phases can be given as follows.

$$
\begin{aligned}
& \text { When } \Omega\left(n^{\beta_{1}}\right)=s=O\left(\left(\frac{n}{\log n}\right)^{\frac{1}{2+\varepsilon_{2}+\varepsilon_{3}}}\right) \text {, then } \\
& t_{\text {Phase } 3}=\frac{s C_{2} C_{3} \frac{n}{s^{1+\varepsilon_{2}+\varepsilon_{3}}}}{\frac{s\left(K_{5}+K_{4} \log n\right)}{K_{6}}}=\frac{K_{6} C_{2} C_{3} \frac{n}{s^{1+\varepsilon_{2}+\varepsilon_{3}}}}{K_{5}+K_{4} \log n}, \\
& t_{\text {total }}=t_{\text {Phase } 1}+t_{\text {Phase } 2}+t_{\text {Phase } 3} \\
& =\frac{K_{6} K_{2} L \frac{n}{s^{1+\varepsilon_{2}+\varepsilon_{3}}}}{K_{4} \log n+K_{5}}+\frac{C_{1} s}{K_{7}}+\frac{K_{6} C_{2} C_{3} \frac{n}{s^{1+\varepsilon_{2}+\varepsilon_{3}}}}{K_{5}+K_{4} \log n},
\end{aligned}
$$


and

$$
\begin{aligned}
R_{1}(n) & =\frac{K_{2} L s \frac{n}{s^{1+\varepsilon_{2}+\varepsilon_{3}}}}{\frac{K_{6} K_{2} L \frac{n}{s^{1+\varepsilon_{2}+\varepsilon_{3}}}}{K_{4} \log n+K_{5}}+\frac{C_{1} s}{K_{7}}+\frac{K_{6} C_{2} C_{3} \frac{n}{s^{1+\varepsilon_{2}+\varepsilon_{3}}}}{K_{5}+K_{4} \log n}} \\
& \geq K_{8} s \log n .
\end{aligned}
$$

The lower bound in (13) is correct when $s=$ $O\left(\left(\frac{n}{\log n}\right)^{\frac{1}{2+\varepsilon_{2}+\varepsilon_{3}}}\right)$.

When $s=O\left(n^{\beta_{1}}\right)$, then

$$
\begin{gathered}
t_{\text {Phase } 3}=\frac{s C_{2} C_{3} \frac{n}{n^{\left(1+\varepsilon_{2}\right) \beta_{1}}}}{\frac{s\left(K_{5}+K_{4} \log n\right)}{K_{6}}}=\frac{K_{6} C_{2} C_{3} \frac{n}{n^{\left(1+\varepsilon_{2}\right) \beta_{1}}}}{K_{5}+K_{4} \log n}, \\
t_{\text {total }}=t_{\text {Phase } 1}+t_{\text {Phase } 2}+t_{\text {Phase } 3} \\
=\frac{K_{6} K_{2} L \frac{n}{n^{\left(1+\varepsilon_{2}\right) \beta_{1}}}}{K_{4} \log n+K_{5}}+\frac{C_{1} s}{K_{7}}+\frac{K_{6} C_{2} C_{3} \frac{n}{n^{\left(1+\varepsilon_{2}\right) \beta_{1}}}}{K_{5}+K_{4} \log n},
\end{gathered}
$$

and

$$
\begin{aligned}
R_{1}(n) & =\frac{K_{2} L s \frac{n}{n^{\left(1+\varepsilon_{2}\right) \beta_{1}}}}{\frac{K_{6} K_{2} L \frac{n}{n\left(1+\varepsilon_{2}\right) \beta_{1}}}{K_{4} \log n+K_{5}}+\frac{C_{1} s}{K_{7}}+\frac{K_{6} C_{2} C_{3} \frac{n}{n^{\left(1+\varepsilon_{2}\right) \beta_{1}}}}{K_{5}+K_{4} \log n}} \\
& \geq K_{8} s \log n .
\end{aligned}
$$

The lower bound in (16) is correct when $s=O\left(\frac{n^{1-\left(1+\varepsilon_{2}\right) \beta_{1}}}{\log n}\right)$.

For the rest of this section, we use the Three-Phase communication with slight modifications based on the value of $s$. Therefore, we only mention the differences between the cases and the Three-Phase scheme that we described above.

2) The Case of: $\Omega\left(\left(\frac{n}{\log n}\right)^{\frac{1}{2+\varepsilon_{2}+\varepsilon_{3}}}\right)=s=$ $O\left(\left(\frac{n \log n}{\log \log n}\right)^{\frac{1}{2}}\right)$

Phase 1: The only differences in this region is the fact that we divide the network into $s$ clusters which results in $\Theta\left(\frac{\log s}{\log \log s}\right)$ sources for each cluster. Similar to Theorem 4.4, it can be proved that there are $\Theta\left(\frac{n}{s}\right)$ nodes in each cluster and all these nodes will be used as relays unlike previous section that we only used nodes inside a circle. The following theorem can be proved for this phase.

Theorem 4.7: The link capacity between any two nodes in a cluster under Phase One and Phase Three of this capacity region is at least $\Theta(1)$.

The proof of this theorem is omitted due to page limitations. The aggregate throughput in this phase is $\Theta(s)=K_{11} s$. Therefore, the time needed in this phase is given by

$$
t_{\text {Phase } 1}^{\prime} \leq \frac{s K_{9} \frac{\log s}{\log \log s} K_{10} L\left(\frac{n}{s}\right)}{K_{11} s}=\frac{K_{9} K_{10} L n \frac{\log s}{\log \log s}}{K_{11} s} .
$$

Phase 2: This phase is also identical to previous one except that the aggregate throughput is $K_{12}\left(\frac{n}{s}\right)$ for transmitting $C_{4} \frac{n}{s}$ symbols. Therefore, the required time for this phase is

$$
t_{\text {Phase 2 }}^{\prime}=\frac{s C_{4}\left(\frac{n}{s}\right)}{K_{12}\left(\frac{n}{s}\right)}=\frac{C_{4} s}{K_{12}} .
$$

Phase 3: This phase is similar to previous one except that the aggregate throughput is $K_{11} s$ and the total required time for this phase can be easily derived as

$$
t_{\text {Phase } 3}^{\prime} \leq \frac{s K_{9} \frac{\log s}{\log \log s} C_{5} C_{3}\left(\frac{n}{s}\right)}{K_{11} s}=\frac{K_{9} C_{5} C_{3} n \frac{\log s}{\log \log s}}{K_{11} s} .
$$

Therefore, the aggregate throughput in this region of $s$ is

$$
R_{2}(n) \geq \frac{s K_{10} L\left(\frac{n}{s}\right)}{\frac{K_{9} K_{10} L n \frac{\log s}{\log \log s}}{K_{11} s}+\frac{C_{4} s}{K_{12}}+\frac{K_{9} C_{5} C_{3} n \frac{\log s}{\log \log s}}{K_{11} s}} .
$$

When $s=O\left(\left(\frac{n \log n}{\log \log n}\right)^{\frac{1}{2}}\right)$, then the first and third terms in the denominator are the dominant factors. Hence, the rate can be written as

$$
\begin{aligned}
R_{2}(n) & \geq \frac{s K_{10} L\left(\frac{n}{s}\right)}{2\left(\frac{K_{9} K_{10} L \frac{\log s}{\log \log s}}{K_{11}}+\frac{K_{9} C_{5} C_{3} \frac{\log s}{\log \log s}}{K_{11}}\right) \frac{n}{s}}, \\
& =K_{13} \frac{s \log \log s}{\log s} .
\end{aligned}
$$

The Three-Phase approach that was described in Section IV-A1 is optimum for that region. However if we use this approach for the second capacity region, it will reduce the capacity from the peak of first capacity region. Now the question is that if we use Section IV-A1 scheme in this region, at what point the throughput capacity for the two schemes are equal, i.e. $\Theta\left(R_{1}(n)\right)=\Theta\left(R_{2}(n)\right)$ ? It turns out that when $\Omega\left(\left(\frac{n}{\log n}\right)^{\frac{1}{2+\epsilon_{2}+\epsilon_{3}}}\right)=s$, then $R_{1}(n)$ can be approximated as $R_{1}(n)=\Theta\left(\frac{n}{s^{1+\varepsilon_{2}+\varepsilon_{3}}}\right)$. For the same capacity region, it is easy to show that $R_{2}(n)=$ $\Theta\left(\frac{s \log \log n}{\log n}\right)^{3}$. By making the two rates $R_{1}(n)$ and $R_{2}(n)$ equal, we arrive at $s=\Theta\left(\left(\frac{n \log n}{\log \log n}\right)^{\frac{1}{2+\varepsilon_{2}+\varepsilon_{3}}}\right)$. Therefore when $\Omega\left(\left(\frac{n}{\log n}\right)^{\frac{1}{2+\varepsilon_{2}+\varepsilon_{3}}}\right)=s=O\left(\left(\frac{n \log n}{\log \log n}\right)^{\frac{1}{2+\varepsilon_{2}+\varepsilon_{3}}}\right)$, we use the transmission scheme shown in Section IV-A1, and for the rest of second capacity region, we utilize the second Three-Phase approach that we explained here. By doing this, the maximum throughput capacity is achieved in the second capacity region.

3) The Case of: $\Omega\left(\left(\frac{n \log n}{\log \log n}\right)^{\frac{1}{2}}\right)=s=O(n)$

For this region, given that the number of source-destination pairs is large, the original Hierarchical MIMO cooperation scheme of [4] provides the highest throughput capacity. The main feature of the Hierarchical Cooperation scheme is the fact that each cluster is further divided into smaller clusters and the distributed MIMO system is utilized in a hierarchical fashion.

Let $A_{s}$ denote an area of a cluster. From Theorem 4.4, it can be shown that the number of sources in each cluster is

${ }^{3}$ we used the fact that in this region, $s=n^{\gamma}$ for some constant value of $\gamma$. 
$M_{s}=\Theta\left(A_{s} s\right)=K_{14} A_{s} s$ and the total number of nodes in each cluster is $N_{s}=\Theta\left(\frac{M_{s} n}{s}\right)=K_{15}\left(\frac{M_{s} n}{s}\right)$ as long as the following condition is satisfied.

$$
A_{s}=\omega\left(\frac{A}{s}\right)
$$

Note that the communication scheme for each hierarchy is very similar to that of the previous section. Due to page limitations, we only state the differences in each communication phase.

Phase 1: This phase is identical to Phase 1 in the previous section, except that there are $K_{14} A_{s} s$ source nodes and $K_{15}\left(\frac{M_{s} n}{s}\right)$ nodes in each cluster. Besides, each node transmits $K_{15}\left(\frac{M_{s}^{s} n}{s}\right)$ blocks of bits to relays and the link throughput is $K_{16} M_{s}^{b}$ where $b$ is a constant between zero and one related to the number of hierarchies. The time required for this phase is

$$
t_{\text {Phase } 1}^{\prime \prime}=\frac{M_{s} \cdot K_{6} K_{15} L\left(\frac{M_{s} n}{s}\right)}{K_{16} M_{s}^{b}} .
$$

Phase 2: This phase is also similar to that in the previous section, except that the total number of transmitted bits in one cluster for each source is $C_{6}\left(\frac{M_{s} n}{s}\right)$ and the aggregate throughput for the network is $K_{17}\left(\frac{M_{s} n}{s}\right)$ symbols. The required time to finish this phase is

$$
t_{\text {Phase 2 }}^{\prime \prime}=\frac{s C_{6}\left(\frac{M_{s} n}{s}\right)}{K_{17}\left(\frac{M_{s} n}{s}\right)}=\frac{C_{6}}{K_{17}} s .
$$

Phase 3: This phase is similar to Phase 3 in the previous section with link throughput in the network as $K_{16} M_{s}^{b}$. The required time to complete this phase is

$$
t_{\text {Phase } 3}^{\prime \prime}=\frac{M_{s} \cdot K_{6} C_{7} C_{3}\left(\frac{M_{s} n}{s}\right)}{K_{16} M_{s}^{b}} .
$$

Thus, the total required time is

$$
t_{\text {total }}^{\prime \prime}=\frac{M_{s} \cdot K_{6} K_{15} L\left(\frac{M_{s} n}{s}\right)}{K_{16} M_{s}^{b}}+\frac{C_{6}}{K_{17}} s+\frac{M_{s} \cdot K_{6} C_{7} C_{3}\left(\frac{M_{s} n}{s}\right)}{K_{16} M_{s}^{b}}
$$

Then, the aggregate throughput for the network is

$$
R_{3}(n)=\frac{s \cdot K_{15} L\left(\frac{M_{s} n}{s}\right)}{t_{\text {total }}^{\prime \prime}}=\frac{K_{18} M_{s} n s}{K_{19} M_{s}^{2-b} n+K_{20} s^{2}},
$$

where $K_{18}=K_{15} L, K_{19}=\frac{K_{6} K_{15} L}{K_{16}}+\frac{K_{6} C_{7} C_{3}}{K_{16}}$ and $K_{20}=$ $\frac{C_{6}}{K_{1} 7}$.

By computing the derivative of $R_{3}(n)$ with respect to $M_{s}$ and equating it to zero, we have

$$
M_{s}=\left(\frac{K_{20}}{K_{19}(1-b)}\right)^{\frac{1}{2-b}}\left(\frac{s^{2}}{n}\right)^{\frac{1}{2-b}}=K_{21}\left(\frac{s^{2}}{n}\right)^{\frac{1}{2-b}},
$$

where $K_{21}=\left(\frac{K_{20}}{K_{19}(1-b)}\right)^{\frac{1}{2-b}}$.

It can be shown from Eq. (28) that the number of sources in each cluster is at least a constant value when $s=\omega(\sqrt{n})$, which guarantees that the number of clusters is less than the number of sources and fulfills the condition in Eq. (22). Therefore, the aggregate throughput is given by

$$
\begin{aligned}
R_{3}(n) & =\frac{K_{18}\left(K_{21}\left(\frac{s^{2}}{n}\right)^{\frac{1}{2-b}}\right) n s}{K_{19}\left(K_{21}\left(\frac{s^{2}}{n}\right)^{\frac{1}{2-b}}\right)^{2-b} n+K_{20} s^{2}}, \\
& =\frac{K_{18} K_{21}}{K_{19} K_{21}^{2-b}+K_{20}} n^{\frac{1-b}{2-b}} s^{\frac{b}{2-b}} \stackrel{(a)}{=} K_{22} s^{\frac{1}{2-b}}\left(\frac{n}{s}\right)^{\frac{1-b}{2-b}} \\
& =K_{22} s^{\frac{1}{2-b}+\log _{s}\left(\frac{n}{s}\right)^{\frac{1-b}{2-b}}}=K_{22} s^{\left(\frac{1}{2-b}+\frac{1-b}{2-b} \log _{s}\left(\frac{n}{s}\right)\right)} \\
& \stackrel{(b)}{=} K_{22} s^{1-\varepsilon_{1}+\varepsilon_{4}} .
\end{aligned}
$$

Equality (a) in the above equation is derived by defining $K_{22}=\frac{K_{18} K_{21}}{K_{19} K_{21}^{2-b}+K_{20}}$ and equality (b) is obtained by letting $\varepsilon_{1}=\frac{1-b}{2-b}$ and $\varepsilon_{4}=\frac{1-b}{2-b} \log _{s}\left(\frac{n}{s}\right)$. It is easy to show that $\varepsilon_{4} \leq \varepsilon_{1}$ in all cases. Note that the result from this scheme is similar to that of [4] when $s=n$. As in [4], if the capacity in the current hierarchy is $K_{22} s^{b}$, then the capacity in the next hierarchy is $K_{22} s^{\left(\frac{1}{2-b}+\frac{1-b}{2-b} \log _{s}\left(\frac{n}{s}\right)\right)}$, which can be shown to increase monotonically. Now we investigate the case when the maximum capacity is achieved or equivalently,

$$
\frac{1}{2-b}+\frac{1-b}{2-b} \log _{s}\left(\frac{n}{s}\right)=1 .
$$

This equality is satisfied when $s=\sqrt{n}$ or $b=1$. However $s=$ $\sqrt{n}$ is not acceptable, because it violates the condition in Eq. (22). In addition, $b$ is always smaller than one and, therefore, the capacity of phase three cannot reach its maximum of $\Theta(s)$.

Now the question is for what value of $s$ we have $\Theta\left(R_{2}(n)\right)=\Theta\left(R_{3}(n)\right)$ in this capacity region. Following a similar procedure as in the previous section, it can be proved that $s=\Theta\left(n^{\frac{1}{2-\varepsilon_{1}+\varepsilon_{4}}}\right)$. Similarly, this capacity region can be divided into two regions. When $\Omega\left(\left(\frac{n \log n}{\log \log n}\right)^{\frac{1}{2}}\right)=$ $s=O\left(n^{\frac{1}{2-\varepsilon_{1}+\varepsilon_{4}}}\right)$, then $R_{2}(n)$ provides a higher throughput capacity of $K_{13}^{\prime} \frac{n}{s}$ and for $\left(n^{\frac{1}{2-\varepsilon_{1}+\varepsilon_{4}}}\right)=s=O(n), R_{3}(n)$ gives $K_{22} s^{1-\varepsilon_{1}+\varepsilon_{4}}$ throughput capacity.

\section{B. Capacity Analysis for Data-Gathering Traffic}

This section is dedicated to computation of achievable capacity for Many-to-One Traffic. We assume that a bandwidth of $W_{2}=W-W_{1}$ is allocated to this traffic. Our analysis is similar to the method used in [10], with the exception that nodes are uniformly distributed in a square plane in this paper as opposed to sphere in [10]. It can be proved that the upper bound is also $\Theta(\log n)$, which is similar to the lower bound.

We adopt a Two-Phase scheme that utilizes $\eta_{4} P_{2}$ and $\left(1-\eta_{4}\right) P_{2}$ Watts for power consumption in Phases 1 and 2 , respectively.

Phase 1. Broadcasting Transmission: In the first phase, only one of the source nodes broadcast its information to the nodes of radius $r$ around it. If $r$ is small enough, all the nodes in the circle with radius $r$ can decode the information. The 
aggregate throughput we can achieve is given by the following theorem.

Theorem 4.8: Let $r=n^{-\varepsilon_{5}}$, where $\varepsilon_{5}$ for $0 \leq \varepsilon_{5} \leq 1$. Then with bandwidth $W_{2}$ and total transmit power $\eta_{4} P_{2}$, an aggregate throughput of at least $K_{23} \log n+K_{24}$ can be achieved.

This theorem is proved in Appendix C.

Phase 2. Cooperative Many-to-One Transmission: In the second phase, all the relays within a radius of $r$ transmit the data along with the source node, thus creating a distributed MISO system. The aggregate throughput that can be achieved is given by the following theorem.

Theorem 4.9: The aggregate throughput of the cooperative Many-to-One transmission scheme is at least $K_{25} \log n+K_{26}$. This theorem is proved in Appendix D. Therefore, the total throughput capacity for the data gathering scheme is $R_{\text {Many-to-One }}(n)=K_{27} \log n+K_{28}$ where $K_{27}=$ $\min \left(K_{23}, K_{25}\right)$ and $K_{28}=\min \left(K_{24}, K_{26}\right)$.

The total throughput capacity in the network as a result of these two types of traffics is the summation of their individual rates, i.e., $R(n)=R_{1}(n)+R_{\text {Many-to-One }}(n)$. The result is provided in Theorem 4.2 .

\section{Discussion AND Future WORK}

The first major contribution of this paper is the separation theorem for heterogeneous traffic. This simple theorem states that when there are multiple classes of traffic in the network, a simple way to achieve the maximum order throughput capacity is to allow all nodes in the network to operate on a single traffic class for an assigned bandwidth. This result implies that multiple-radio multiple-channel systems are order-optimum for heterogeneous traffic. The main reason for this result is the fact that nodes that are not part of a specific traffic can be utilized as relays [9], which clearly improve the throughput capacity of the network.

The second major contribution of this paper is the computation of the achievable throughput capacity when the number of relays and source-destination pairs are changing as a function of $n$. Gastpar and Vetterli [9] have solved this problem when there is only one source-destination pair in the network and the rest of the nodes are relays. We have shown different forwarding strategies when the ratio between relays and unicast sessions changes by utilizing an extended version of the threephase approach introduced in [4]. Our results also corroborate previous results obtained in [4] when the number of sourcedestination pairs is $n$ and in [9] when there are $\Theta(1)$ sourcedestination pairs in the network.

Note that, in the last capacity region, the achievable capacity is $n^{1-\varepsilon_{1}+\varepsilon_{4}}$ instead of capacity of $n^{1-\varepsilon_{1}}$ as reported in [4]. The gain of $n^{\varepsilon_{4}}$ for $\varepsilon_{4}=\frac{1-b}{2-b} \log _{s}\left(\frac{n}{s}\right)$ is achieved by employing relays to improve the throughput. This gain reduces as $s$ tends to $n$, because $\varepsilon_{4} \rightarrow 0$.

The unicast capacity for $\Omega\left(S_{2}=\left(\frac{n \log n}{\log \log n}\right)^{\frac{1}{2+\varepsilon_{2}+\varepsilon_{3}}}\right)=$ $s=O\left(S_{3}=\left(\frac{n \log n}{\log \log n}\right)^{\frac{1}{2}}\right)$ is $\Theta\left(\frac{s \log \log s}{\log s}\right)$. However, if the number of sources in each cluster is a constant value instead of a random variable, then it is easy to show that a capacity of $\Theta(s)$ can be achieved.

Fig. 2 plots the capacity region that was derived in (7). From this figure, we see that, when the number of unicast sessions is from 1 to $\Theta\left(S_{1}=\left(\frac{n}{\log n}\right)^{\frac{1}{2+\varepsilon_{2}+\varepsilon_{3}}}\right)$, the majority of nodes are part of Many-to-One Traffic and we call this region as Many-to-One Traffic. The achieved capacity in this region is the optimum value. When $\Omega\left(S_{1}\right)=s=O\left(S_{4}=n^{\frac{1}{2-\varepsilon_{1}+\varepsilon_{4}}}\right)$, then the number of nodes for both traffic patterns are comparable. Hence, we call it Heterogeneous Traffic region. It is not clear whether our achievable capacity region is optimum for this region. Finally when $s=\Omega\left(S_{4}\right)$, then majority of nodes are involved in unicast communication and we call this region Unicast traffic.

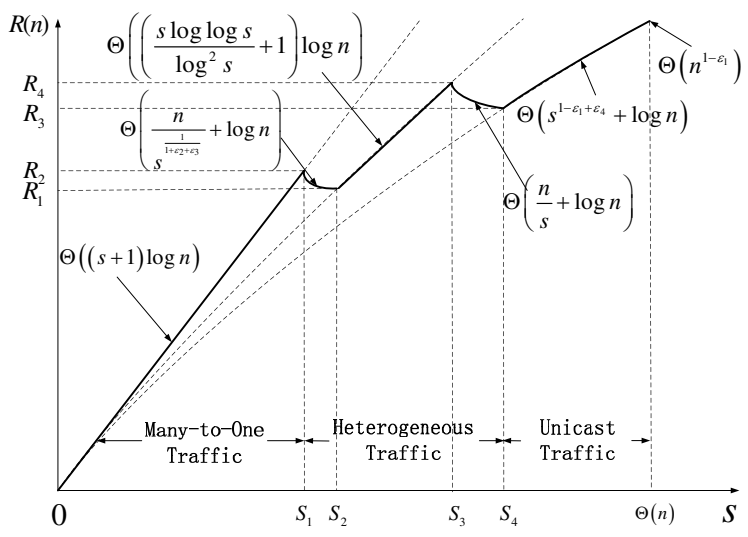

Fig. 2. The achievable aggregate throughput.

It is worthy of note that the capacity actually decreases in two regions as $s$ increases. These two regions particularly require more investigation to find better communication schemes which is the subject of future study.

\section{ACKNOWLEDGEMENT}

Research was partially sponsored by the U.S. Army Research Laboratory under the Network Science Collaborative Technology Alliance, Agreement Number W911NF-09-0053, by Army Research Office under agreement number W911NF05-1-0246, by the National Science Foundation under grant CCF-0729230, and by the Baskin Chair of Computer Engineering. The views and conclusions contained in this document are those of the author(s) and should not be interpreted as representing the official policies, either expressed or implied, of the U.S. Army Research Laboratory or the U.S. Government. The U.S. Government is authorized to reproduce and distribute reprints for Government purposes notwithstanding any copyright notation hereon.

\section{REFERENCES}

[1] P. Gupta and P.R.Kumar, "The capacity of wireless networks," IEEE Transactions on Information Theory, vol. 46, no. 2, pp. 388-404, March 2000 . 
[2] L.-L. Xie and P.R.Kumar, "A network information theory for wireless communication: Scaling laws and optimal operation," IEEE Transactions on Information Theory, vol. 50, no. 5, pp. 748-767, May 2004.

[3] _ _ "On the path-loss attenuation regime for positive cost and linear scaling of transport capacity in wireless networks," IEEE Transactions on Information Theory, vol. 52, no. 6, pp. 2313-2328, June 2006.

[4] A. Ozgur, O. Lévêque, and D. N.C.Tse, "Hierarchical cooperation achieves optimal capacity scaling in ad hoc networks," IEEE Transactions on Information Theory, vol. 55, no. 10, pp. 3549-3572, October 2007.

[5] A. Keshavarz-Haddad and R. Riedi, "Bounds for the capacity of wireless multihop networks imposed by topology and demand," in MobiHoc, September 2007, pp. 256-265.

[6] S. Toumpis, "Asymptotic capacity bounds for wireless networks with non-uniform traffic patterns," IEEE Transactions on Wireless Communication, vol. 7, no. 5, pp. 1-12, May 2008.

[7] B. Liu, D. Towsley, and A. Swami, "Data gathering capacity of large scale multihop wireless networks," in Mobihoc, 2008.

[8] M. Ji, Z. Wang, H. Sadjadpour, and J. J. Garcia-Luna-Aceves, "Capacity of wireless networks with heterogeneous traffic," in Globecom, November 2009.

[9] M. Gastpar and M. Vetterli, "On the capacity of wireless networks: The relay case," in INFOCOM, June 2002, pp. 1577-1586.

[10] H. E. Gamal, "On the scaling laws of dense wireless sensor netowrks: The data gatering channel," IEEE Transactions on Information Theory, vol. 51, no. 3, pp. 1229-1234, March 2005.

[11] D. Marco, E. J. Duarte-Melo, M. Liu, and D. L. Neuhoff, "On the many-to-one transport capacity of a dense wireless sensor network and the compressibility of its data," in IPSN, 2003.

[12] V. Rodoplu and T. H.Meng, "Core capacity of wireless ad hoc networks," in The 5th International Symposium on Wireless Personal Multimedia Communications, September 2002, pp. 247-251.

[13] M. Kyoung and V. Rodoplu, "Core capacity region of portable wireless networks," in Globecom, September 2004, pp. 256-265.

[14] D. Tse and P. Viswanath, Fundamentals of Wireless Communication. Cambridge University Press, 2004.

[15] B. Motwani and P. Raghavan, Randomized Algorithms. Cambridge University Press, 1995.

\section{APPENDIX A \\ PROOF OF THEOREM 4.4}

For the number of source nodes in each cluster, we can consider the problem as bins and balls problem. Here the number of balls is $s$ and the number of bins is $s^{1+\varepsilon_{2}}$. By using Lemma 4.3, the maximum number of source nodes in each cluster is given by

$$
\begin{aligned}
B\left(s, s^{1+\varepsilon_{2}}\right) & =\Theta\left(\frac{\log s^{1+\varepsilon_{2}}}{\log \frac{s^{1+\varepsilon_{2}}}{s}}\right), \\
& =\Theta\left(\frac{\left(1+\varepsilon_{2}\right) \log s}{\varepsilon_{2} \log s}\right)=\Theta(1) .
\end{aligned}
$$

For the number of the nodes in each cluster, we can use Chebychev's inequality [15]. The Chebychev's inequality is given below.

Lemma A.1: Let $X$ be a random variable with mean and standard deviation of $\mu_{x}$ and $\sigma_{x}$ respectively. Then

$$
\operatorname{Pr}\left(\left|X-\mu_{x}\right| \geq \alpha_{x}\right) \leq \frac{\sigma_{x}^{2}}{\alpha_{x}^{2}}
$$

for any any $\alpha_{x}>0$.

Assume $\alpha_{x}^{2}=\sqrt{\alpha \times \frac{n}{s^{1+\varepsilon_{2}}}}$ where $\alpha$ is defined as a sequence such that $\lim _{\frac{n}{s^{1+\varepsilon_{2}}} \rightarrow \infty} \frac{\alpha}{s^{1+\varepsilon_{2}}}=\gamma_{1}$ for any positive value of $\gamma_{1}$. Define the random variable $V_{n}$ as the number of nodes in each cluster. Because the nodes uniformly distributed in the network, then the mean and variance of $V_{n}$ is given by $\mu=\frac{n}{s^{1+\varepsilon_{2}}}$ and $\sigma^{2}=\frac{n}{s^{1+\varepsilon_{2}}}\left(1-\frac{1}{s^{1+\varepsilon_{2}}}\right)$ respectively. By using Chebychev's inequality, we arrive at

$$
\begin{aligned}
\operatorname{Pr}\left(\left|V_{n}-\frac{n}{s^{1+\varepsilon_{2}}}\right| \geq \sqrt{\alpha \times \frac{n}{s^{1+\varepsilon_{2}}}}\right) & \leq \frac{\frac{n}{s^{1+\varepsilon_{2}}}\left(1-\frac{1}{s^{1+\varepsilon_{2}}}\right)}{\alpha \times \frac{n}{s^{1+\varepsilon_{2}}}} \\
& \leq \frac{1}{\alpha}\left(1-\frac{1}{s^{1+\varepsilon_{2}}}\right)
\end{aligned}
$$

The second term on the right hand side of (33) goes to zero as $\frac{n}{s^{1+\varepsilon_{2}}} \rightarrow \infty$. Thus with probability close to one $\left|V_{n}-\frac{n}{s^{1+\varepsilon_{2}}}\right| \leq \sqrt{\alpha \times \frac{n}{s^{1+\varepsilon_{2}}}}$ or equivalently, $V_{n}=\Theta\left(\frac{n}{s^{1+\varepsilon_{2}}}\right)$. Similarly, it can be proved that in a circle with radius of $\frac{\sqrt{A}}{2 s \frac{1+\varepsilon_{2}+\varepsilon_{3}}{2}}$ or $\frac{\sqrt{A}}{2 n \frac{\left(1+\varepsilon_{2}\right) \beta_{1}}{2}}$, the number of nodes is $\Theta\left(\frac{n}{s^{1+\varepsilon_{2}+\varepsilon_{3}}}\right)$ or $\Theta\left(\frac{n}{n^{\frac{\left(1+\varepsilon_{2}\right) \beta_{1}}{2}}}\right)$ respectively.

\section{APPENDIX B}

\section{ProOF OF THEOREM 4.5}

To prove Theorem 4.5, we consider two cases. First, we calculate the aggregate throughput when $\Omega\left(n^{\beta_{1}}\right)=s=$ $O\left(\left(\frac{n}{\log n}\right)^{\frac{1}{2+\varepsilon_{2}+\varepsilon_{3}}}\right)$. Then, we consider the case when $s=$ $O\left(n^{\beta_{1}}\right)$.

A. When $\Omega\left(n^{\beta_{1}}\right)=s=O\left(\left(\frac{n}{\log n}\right)^{\frac{1}{2+\varepsilon_{2}+\varepsilon_{3}}}\right)$

The capacity between a source node $k$ and the relay $i$ is

$$
\begin{aligned}
& C_{k i}^{\text {Case } 1}=W_{1} \log \left(1+\frac{\frac{P_{1}^{\prime}}{s}\left|H_{k i}\right|^{2}}{W_{1} N_{0}+\sum_{j \in T, j \neq k} \frac{P_{1}^{\prime}}{s}\left|H_{j i}\right|^{2}}\right) \\
& =W_{1} \log \left(1+\frac{\frac{P_{1}^{\prime}}{s}\left(\frac{\sqrt{G}}{d_{k i}^{\frac{\alpha}{2}}}\right)^{2}}{W_{1} N_{0}+\sum_{j \in T, j \neq k} \frac{P_{1}^{\prime}}{s}\left(\frac{\sqrt{G}}{d_{j i}^{\frac{\alpha}{2}}}\right)^{2}}\right) \\
& \geq W_{1} \log \\
& \left(1+\frac{\frac{P_{1}^{\prime}}{s}\left(\frac{\sqrt{G}}{\left(\frac{\sqrt{A}}{\frac{1+\varepsilon_{2}+\varepsilon_{3}}{2}}\right)^{\frac{\alpha}{2}}}\right)^{2}}{W_{1} N_{0}+\sum_{l=1}^{\infty} 8 l \frac{P_{1}^{\prime}}{s}\left(\frac{\sqrt{G}}{\left(\left(l \sqrt{K_{6}}-1\right) \frac{\sqrt{A}}{\left.s^{\frac{\left(1+\varepsilon_{2}\right)}{2}}\right)^{\frac{\alpha}{2}}}\right)^{2}}\right.}\right) \\
& =W_{1} \log \\
& \left(1+\frac{\frac{P_{1}^{\prime} G}{A^{\frac{\alpha}{2}}} s^{\frac{\left(1+\varepsilon_{2}+\varepsilon_{3}\right) \alpha}{2}-1}}{W_{1} N_{0}+\left(\frac{P_{1}^{\prime} G}{A^{\frac{\alpha}{2}}} \sum_{l=1}^{\infty} \frac{8 l}{\left(l \sqrt{K_{6}}-1\right)^{\alpha}}\right) s^{\frac{\left(1+\varepsilon_{2}\right) \alpha}{2}-1}}\right) \\
& >W_{1} \log \left(\frac{\frac{P_{1}^{\prime} G}{A^{\frac{\alpha}{2}}} s^{\frac{\left(1+\varepsilon_{2}+\varepsilon_{3}\right) \alpha}{2}-1}}{2\left(\frac{P_{1}^{\prime} G}{A^{\frac{\alpha}{2}}} \sum_{l=1}^{\infty} \frac{8 l}{\left(l \sqrt{K_{6}}-1\right)^{\alpha}}\right) s^{\frac{\left(1+\varepsilon_{2}\right) \alpha}{2}-1}}\right)
\end{aligned}
$$




$$
\begin{aligned}
& =W_{1} \log \left(\frac{1}{2 \sum_{l=1}^{\infty} \frac{8 l}{\left(l \sqrt{K_{6}}-1\right)^{\alpha}}}\right)+W_{1} \log \left(s^{\frac{\varepsilon_{3} \alpha}{2}}\right) \\
& \stackrel{(a)}{\geq} K_{5}+W_{1} \log \left(\left(n^{\beta_{1}}\right)^{\frac{\varepsilon_{3} \alpha}{2}}\right) \\
& =K_{5}+M_{1} \log n
\end{aligned}
$$

where inequality $(a)$ is derived because when $\alpha>2$, then $\sum_{l=1}^{\infty} \frac{8 l}{\left(l \sqrt{K_{6}}-1\right)^{\alpha}}$ converges to a constant value and $M_{1}$ is a positive constant value.

\section{B. When $s=O\left(n^{\beta_{1}}\right)$}

Under the condition it is easy to show that $\frac{\sqrt{A}}{s^{\frac{1+\varepsilon_{2}}{2}}}>$ $\frac{\sqrt{A}}{n \frac{\left(1+\varepsilon_{2}\right) \beta_{2}}{2}}>\frac{\sqrt{A}}{n^{\frac{\left(1+\varepsilon_{2}\right) \beta_{1}}{2}}}$ for appropriate value of $\beta_{2}$ such that $\beta_{2}<\frac{\left[\left(1+\varepsilon_{2}\right) \alpha-2\right]}{\left(1+\varepsilon_{2}\right) \alpha} \beta_{1}$.

If the source node transmits information within a circle of radius $\frac{\sqrt{A}}{2 n \frac{\left(1+\varepsilon_{2}\right) \beta_{1}}{2}}$, then the achievable rate between nodes $k$ and $i$ is given below.

$C_{k i}^{\text {Case } 2}$

$$
=W_{1} \log \left(1+\frac{\frac{P_{1}^{\prime}}{s}\left(\frac{\sqrt{G}}{d_{k i}^{\frac{\alpha}{2}}}\right)^{2}}{W_{1} N_{0}+\sum_{j \in T, j \neq k} \frac{P_{1}^{\prime}}{s}\left(\frac{\sqrt{G}}{d_{j i}^{\frac{\alpha}{2}}}\right)^{2}}\right)
$$

$\geq W_{1} \log$

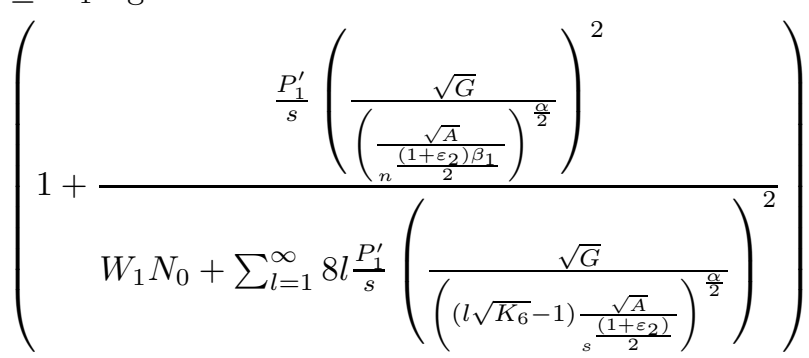

(a)

$\geq W_{1} \log$

$$
\begin{aligned}
& \left(1+\frac{\frac{P_{1}^{\prime}}{n^{\beta_{1}}}\left(\frac{\sqrt{G}}{\left(\frac{\sqrt{A}}{\frac{\left(1+\varepsilon_{2}\right) \beta_{1}}{2}}\right)^{\frac{\alpha}{2}}}\right)^{2}}{W_{1} N_{0}+\sum_{l=1}^{\infty} 8 l \frac{P_{1}^{\prime}}{1}\left(\frac{\sqrt{G}}{\left(\left(l \sqrt{K_{6}}-1\right) \frac{\sqrt{A}}{\frac{\left(1+\varepsilon_{2}\right) \beta_{2}}{2}}\right)^{\frac{\alpha}{2}}}\right)^{2}}\right) \\
& =W_{1} \log \left(1+\frac{\frac{P_{1}^{\prime} G}{A^{\frac{\alpha}{2}}} n^{\frac{\left(1+\varepsilon_{2}\right) \alpha \beta_{1}}{2}-\beta_{1}}}{W_{1} N_{0}+\left(\frac{P_{1}^{\prime} G}{A^{\frac{\alpha}{2}}} \sum_{l=1}^{\infty} \frac{8 l}{\left(l \sqrt{K_{6}}-1\right)^{\alpha}}\right) n^{\frac{\left(1+\varepsilon_{2}\right) \alpha \beta_{2}}{2}}}\right) \\
& >W_{1} \log \left(\frac{\frac{P_{1}^{\prime} G}{A^{\frac{\alpha}{2}}} n^{\frac{\left(1+\varepsilon_{2}\right) \alpha \beta_{1}}{2}}-\beta_{1}}{2\left(\frac{P_{1}^{\prime} G}{A^{\frac{\alpha}{2}}} \sum_{l=1}^{\infty} \frac{8 l}{\left(l \sqrt{K_{6}}-1\right)^{\alpha}}\right) n^{\frac{\left(1+\varepsilon_{2}\right) \alpha \beta_{2}}{2}}}\right) \\
& =W_{1} \log \left(\frac{1}{2 \sum_{l=1}^{\infty} \frac{8 l}{(2 l-1)^{\alpha}}}\right)+W_{1} \log \left(n^{\frac{\left(1+\varepsilon_{2}\right) \alpha\left(\beta_{1}-\beta_{2}\right)}{2}-\beta_{1}}\right) \\
& =K_{5}+M_{2} \log n
\end{aligned}
$$

(a) is derived by replacing distance for interference with smaller distance and replacing $s$ in numerator by its maximum value and replace it with 1 in the denominator. Note that $M_{2}=W_{1}\left(\frac{\left(1+\varepsilon_{2}\right) \alpha\left(\beta_{1}-\beta_{2}\right)}{2}-\beta_{1}\right)$ is a positive value given the condition above for $\beta_{2}$.

Thus, the achievable rate between nodes $k$ and $i$ is given by

$$
\begin{aligned}
R_{k i}(n) & =\min \left(C_{k i}^{\text {Case 1 }}, C_{k i}^{\text {Case 2 }}\right), \\
& =\min \left(M_{1} \log n+K_{5}, M_{2} \log n+K_{5}\right), \\
& =\min \left(M_{1}, M_{2}\right) \log n+K_{5}, \\
& =K_{4} \log n+K_{5}
\end{aligned}
$$

where $K_{4}=\min \left(M_{1}, M_{2}\right)$.

Given the TDMA parameter $K_{6}$, there are on average $\frac{s}{K_{6}}$ nodes sending their information. Thus, the aggregate throughput is given by

$$
R_{\text {Phase } 1}(n) \geq \frac{s\left(K_{4} \log n+K_{5}\right)}{K_{6}} .
$$

\section{APPENDIX C}

\section{PROOF OF THEOREM 4.8}

Under the Many-to-One transmission model, the capacity between source node $k$ and the relay node $i$ in the circle with radius $r=n^{-\varepsilon_{5}}$ is given by

$$
\begin{aligned}
C_{k i} & =W_{2} \log \left(1+\frac{\eta_{4} P_{2}\left|H_{k i}\right|^{2}}{W_{2} N_{0}}\right) \\
& \geq W_{2} \log \left(1+\frac{\eta_{4} P_{2}\left(\frac{\sqrt{G}}{r^{\frac{\alpha}{2}}}\right)^{2}}{W_{2} N_{0}}\right) \\
& =W_{2} \log \left(1+\frac{\eta_{4} P_{2} G}{W_{2} N_{0}} n^{\varepsilon_{5} \alpha}\right) \geq W_{2} \log \left(\frac{\eta_{4} P_{2} G}{W_{2} N_{0}} n^{\varepsilon_{5} \alpha}\right) \\
& =W_{2} \log \left(\frac{\eta_{4} P_{2} G}{W_{2} N_{0}}\right)+W_{2} \varepsilon_{5} \alpha \log (n)=K_{24}+K_{23} \log n
\end{aligned}
$$

where $K_{23}=W_{2} \varepsilon_{5} \alpha$ and $K_{24}=W_{2} \log \left(\frac{\eta_{4} P_{2} G}{W_{2} N_{0}}\right)$.

\section{APPENDIX D}

\section{PROOF OF THEOREM 4.9}

Similar to Theorem 4.4, we can prove that the number of nodes in the circle with radius of $n^{-\varepsilon_{5}}$ is $\Theta\left(n^{1-2 \varepsilon_{5}}\right)=$ $M_{4} n^{1-2 \varepsilon_{5}}$. Then the capacity is computed as

$$
\begin{aligned}
C_{\text {Many-to-One }} & =W_{2} \log \left(1+\frac{\left(1-\eta_{4}\right) P_{2} \sum_{j=1}^{M_{4} n^{1-2 \varepsilon_{5}}}\left(\frac{\sqrt{G}}{d_{j}^{\frac{\alpha}{2}}}\right)^{2}}{W_{2} N_{0}}\right) \\
& \geq W_{2} \log \left(\frac{\left(1-\eta_{4}\right) P_{2} G M_{4}}{W_{2} N_{0} A^{\frac{\alpha}{2}}} n^{1-2 \varepsilon_{5}}\right) \\
& =K_{25} \log n+K_{26}
\end{aligned}
$$

where $K_{25}=1-2 \varepsilon_{5}$ and $K_{26}=W_{2} \log \left(\frac{\left(1-\eta_{4}\right) P_{2} M_{4}}{W_{2} N_{0} A^{\frac{\alpha}{2}}}\right)$. 